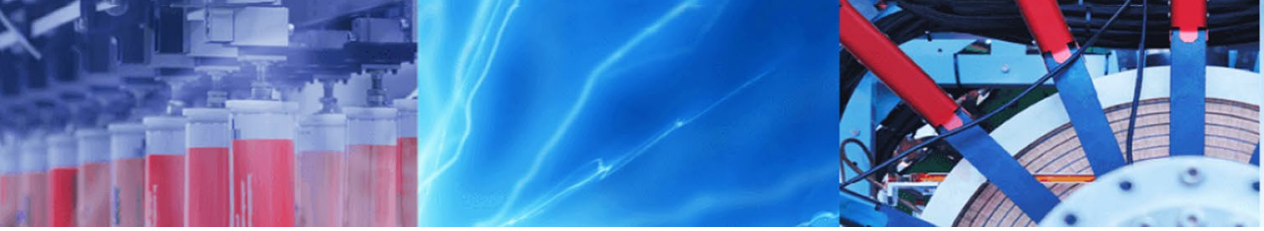

Research Article

\title{
Identifying redundant features using unsupervised learning for high-dimensional data
}

\author{
Asir Antony Gnana Singh Danasingh ${ }^{1}$ [ $\cdot$ Appavu alias Balamurugan Subramanian ${ }^{2} \cdot$ Jebamalar Leavline Epiphany $^{3}$
}

Received: 17 February 2020 / Accepted: 29 June 2020 / Published online: 11 July 2020

(c) Springer Nature Switzerland AG 2020

\begin{abstract}
In the digital era, classifiers play a vital role in various machine learning applications such as medical diagnosis, weather prediction and pattern recognition. The classifiers are built by classification algorithms using data. Nowadays, the data are high dimensional in nature since the data are massively generated due to advancements in information and communication technology. The high-dimensional space contains irrelevant and redundant features; both of them reduce the classification accuracy and increase space and building time of the classifiers. Redundancy and relevancy analysis mechanisms of the feature selection process remove the irrelevant and redundant features. Identifying the irrelevant features is a simple task since that only considers the relevancy between each feature and the target class of a dataset using any one of the statistical or information theoretic measures. Identifying the redundant features from a dataset is quite difficult, especially in high-dimensional space since it needs to consider the relevancy among the features. This leads to more computational complexity and an inappropriate relevancy measure that can degrade the classification accuracy. In order to overcome these problems, this paper presents an unsupervised learning-based redundancy analysis mechanism for feature selection by evaluating various clustering techniques in terms of average redundancy rate and runtime.
\end{abstract}

Keywords Clustering $\cdot$ Redundancy rate $\cdot \mathrm{K}$-means clustering $\cdot$ EM clustering $\cdot$ Hierarchical clustering

\section{Introduction}

In the digital era, data are being generated massively in all the areas including the medical, engineering, technology, science, economics, etc. [1], due to the advancements in information and communicating engineering. Nowadays, classifying the data for making decisions and analysis is a very challenging task. In general, classification is carried out using the classifier that is developed using a classification algorithm with data [2]. These massively generated data are high dimensional as they contain more features. The high-dimensional data are redundant and irrelevant in nature. These, irrelevant and redundant features increase the building time of the classifier and reduce the classification accuracy [3]. Therefore, feature selection is designed with relevancy and redundancy analysis mechanisms for removing the irrelevant and redundant features from a high-dimensional space [4]. Identifying the relevant features is a simple task since it measures only the relevancy between each feature and the target class using any one of the statistical or information theoretic measures. Thus, the relevancy analysis is effortless [5].

Identifying the redundant features in a high-dimensional space is quite complex since the feature selection method needs a special mechanism to identify the relevancy among the features. Therefore, redundancy analysis takes more computations than the relevancy analysis and it needs a suitable relevancy measure. In order to

Asir Antony Gnana Singh Danasingh, asirantony@gmail.com; Appavu alias Balamurugan Subramanian, app_s@yahoo.co.in; Jebamalar Leavline Epiphany, jebilee@gmail.com | ${ }^{1}$ Department of Computer Science and Engineering, Anna University, BIT Campus, Tiruchirappalli, India. ${ }^{2}$ Department of Information Technology, K.L.N College of Information Technology, Pottapalayam, Sivagangai, India. ${ }^{3}$ Department of Electronics and Communication Engineering, Anna University, BIT Campus, Tiruchirappalli, India. 
overcome these problems in the redundancy analysis phase of the feature selection process, the clustering techniques can be employed.

Thus, identifying a suitable clustering technique is a challenging task in the field of machine learning and feature selection. This paper presents an empirical assessment on various clustering techniques for redundancy analysis in feature selection for high-dimensional data classification in terms of average redundancy rate and runtime.

\section{Clustering techniques}

Clustering is a process of grouping objects into number of clusters based on their similarity. The intra-cluster objects are highly similar than the inter-cluster objects. Clustering can be carried out using various clustering algorithms with different clustering techniques. These clustering techniques are used to solve numerous real-world problems. In medical imaging, clustering is used in the analysis of antimicrobial activity and intensity-modulated radiation therapy (IMRT) segmentation. In business and marketing, clustering models are used to group the shopping items and to conduct market research. In World Wide Web (WWW), clustering is employed for social network analysis, search result grouping, etc.

In computer science, clustering is employed in software evolution, image segmentation, evolutionary algorithms, recommender systems, Markov chain, Monte Carlo methods and anomaly detection. In social science, clustering techniques are applied in crime analysis, educational data mining, typologies, field robotics, mathematical chemistry, climatology, petroleum geology, physical geography, etc. [6]. In feature selection, clustering techniques are used to group the similar features in order to remove the redundant features from the dataset to improve the accuracy of the classifier.

Usually, the clustering algorithms develop the clustering models with data. The clustering models are employed to identify the group of an ungrouped object. The clustering models can be categorized into various types such as connectivity-based model, centroid-based model and distribution-based models. In connectivity-based model, the model is built based on the distance connectivity. This model can be termed as hierarchical clustering (HC). In centroid-based model, each cluster can be represented using mean vectors. The well-known K-means clustering $(\mathrm{KM})$ is of this category.

In distribution-based model, clusters can be modeled using any one of the statistical distributions, such as multivariate normal distributions. Expectation-maximization clustering (EC) [7] is a typical example of this category. In this paper, centroid model-based K-means clustering, distribution model-based expectation maximization clustering and connectivity model-based hierarchical clustering are evaluated for redundancy analysis in feature selection.

\subsection{K-means clustering (KC)}

In this clustering model, $K$ features from the dataset are randomly chosen as initial cluster-centers, and then similarity between each feature and each cluster-center is calculated using a distance measure. As a result, each feature is assigned to a cluster which has the maximum feature to cluster-center similarity. Individual features are assigned to a different cluster-center if the reassignment decreases the sum of within-cluster similarities and sumof-squares of feature to cluster-center similarities. Average of the features is computed in each cluster to obtain $K$ new cluster-center locations until there is no change in cluster assignments or the stopping criteria are met.

The features of the training dataset are grouped based on the cluster-center of each cluster using Euclidian distance similarity measure. The cluster-center $\omega$ is refined in an iterative fashion to form $K$ perfect clusters of features using Eq. (1).

$W(\omega)=\frac{1}{2} \sum_{k=1}^{K} \sum_{\omega(i)=k^{\prime}} \sum_{\omega(j)=k}\left\|X_{i}^{\prime}-X_{j}^{\prime}\right\|^{2}=\sum_{k=1}^{K} N_{k}^{\prime} \sum_{\omega(i)^{\prime}=k}\left\|X_{i}^{\prime}-m_{k}^{\prime}\right\|^{2}$

where $X_{1}, \ldots, X_{N}$ are the features of the training dataset. Each feature of the dataset is assigned to any one of the $K$ clusters. $\omega(i)$ indicates the cluster index of the $i$ th feature. $m_{k}$ denotes the mean vector of the $k$ th cluster. $N_{k}$ denotes the number of features in the $k$ th cluster. $W(\omega)$ is the Euclidian distance measure with respect to the clustercenter [8].

\subsection{Expectation maximization clustering (EC)}

In expectation maximization clustering, the Gaussian probability distribution is used to group the features to form the clusters. This method consists of three steps, namely initialization, expectation and maximization.

Let $D$ be the input dataset, $K$ be the total number of clusters, $n$ be the maximum number of iterations, and e be the accepted error to converge. For each iteration, the 
expectation (Step 2) followed by maximization (Step 3) is performed until convergence or the maximum number of iterations [9].

Step1: Initialization

Let $k$ be the index of a cluster such that $k=1,2, \ldots, K$ where $K$ is the total number of clusters. The parameter vector $\theta$ is calculated using the mean $\left(\mu_{k}\right)$ and the covariance matrix $P_{k}(t)$ as given in Eq. (2) where $P_{k}(t)$ denotes the features of the Gaussian probability distribution that is employed to identify the observed and unobserved feature points of the dataset $D$.

$\theta(t)=\left(\mu_{k}(t), P_{k}(t)\right), \quad k=1, \ldots, K$

$t=0$ represents an initial instant. This means that the initial values for $\mu_{k}$ and $P_{k}(t)$ are randomly generated. The main objective of EC algorithm is to approximate the parameter vector $\theta$ of the real distribution.

Step 2: Expectation

In this step, the probability of elements $P\left(\omega_{k} \mid X_{j}\right)$ of each cluster $\left(\omega_{k}\right)$ is estimated as in Eq. (3) where $n$ is the number of iterations and $e$ is the accepted error to converge. Each element is developed using a feature vector $X_{i}$. The level of relevancy among the features in each cluster $\omega_{k}$ is evaluated using the likelihood of each feature present in the cluster.

$$
P\left(\omega_{k} \mid X_{i}\right)=\frac{\left|\sum_{k}(t)\right|^{-1 / 2} e^{n_{k}} P_{k}(t)}{\sum_{k=1}^{K}\left|\sum_{k}(t)\right|^{-1 / 2} e^{n_{k} P_{k}(t)}}
$$

Step 3: Maximization

In this step, the parameters of the probability distribution of each cluster for the next step are estimated [Eq. (4)]. The mean $\mu_{k}$ of cluster $k$ can be calculated through the mean of all feature points based on the level of relevancy of each feature point.

$\mu_{k}(t+1)=\frac{\sum_{i=1}^{N} P\left(\omega_{k} \mid X_{i}\right) X_{i}}{\sum_{i=1}^{N} P\left(\omega_{k} \mid X_{i}\right)}$

Bayes theorem $P(A \mid B)=P(B \mid A) \times P(A) P(B)$ is used to calculate the covariance matrix for the next iteration, and the cluster occurrence [Eq. (5)] is computed depending on the conditional probabilities.

$$
\sum_{k}(t+1)=\frac{\sum_{i=1}^{N} P\left(\omega_{k} \mid X_{i}\right)\left(X_{i}-\mu_{k}(t)\right)\left(X_{i}-\mu_{k}(t)\right)}{\sum_{i=1}^{N} P\left(\omega_{k} \mid X_{i}\right)}
$$

The probability of occurrence of each cluster is calculated using the mean of probabilities $\omega_{k}$ as a function of the level of relevancy of each feature point from the cluster-center as in Eq. (6).

$P_{k}(t+1)=\frac{1}{2} \sum_{i=1}^{N} P\left(\omega_{k} \mid X_{i}\right)$

The features represent the parameter vector $\theta$ that determines the probability distribution of each cluster that can be used in the next iteration of the algorithm.

\subsection{Hierarchical clustering (HC)}

In this method, clusters are formed using the distancebased connectivity. These hierarchal clustering techniques can be categorized as top-down and bottom-up models based on how the connections are made with split and merge to form the group of objects or features in a tree form. Top-down approach is also known as divisive method in which the original features are split recursively one move down to form the hierarchy based on their similarity with a linkage method. The bottom-up approach is also known as agglomerative method. In this method, each feature initiates in its own group and the pair of groups are merged toward upward direction to form the hierarchical cluster using the similarity measure and linkage function. The linkage function can form the hierarchical cluster using the distance measures [10]. The distance measures are used to find the similarity between the features based on their distance. If $X_{i}$ and $X_{j}$ are two features where $i \neq j$, the distance $d$ between these two features is defined as $d_{i, j}^{2}=\left(X_{i}-X_{j}\right)\left(X_{i}-X_{j}\right)^{\prime}$ where $i \neq j$.

\section{Redundancy analysis in industrial applications}

In the recent past, data are being generated massively in all the industries including medical, banking, agriculture, education, construction, social media, etc. [1]. Due to the growth of the information technology and artificial intelligence, the data-driven decisions are taken in most of the industrial process from acquiring the raw materials to market the finished goods or services. In general, the classifier is employed to make data-driven decisions in industries. The classifier is built using the classification algorithm with the high-dimensional data [11-13]. The high-dimensional raw industrial data contain the irrelevant and redundant features that reduce accuracy of the classifiers and building time of the classifier. Hence, relevancy and redundancy analysis mechanisms are required to remove the irrelevant and redundant features from the data. Relevancy analysis is a simple task than the redundancy analysis [5]. The 
redundancy analysis contributes significantly for developing classifiers for various industrial applications. In computer network industry, this is used for developing antivirus and intrusion detection systems [14, 15]. Transport industry requires the forecasting of number of passengers [16]. In banking sector, it is inevitable in loan approval prediction [17] and fraudulent detection [18]. The medical field uses classification for disease diagnosis [19]. It is also adopted in automated job suggestion for recruitment [20]. In agriculture field, crop yield prediction [21], plant disease detection [22], leaf disease detection [23], soil classification, soil fertility prediction, etc., are based on classifiers [24]. In software engineering, software fault detection $[25,26]$ employs classifiers. In metrology, there are many applications including weather prediction, cyclone, storm and rainfall forecasting [27]. Bearing fault diagnosis is one of the typical applications in mechanical industries [28]. Some other industrial applications include face recognition [29], speech recognition [30, 31], document classification [32,33] and vehicle detection [34].

\section{Proposed method for identifying suitable clustering mechanism for redundancy analysis}

Statement 1 If a clustering method produces higher average intra-cluster correlation on a dataset, then the clusters formed are highly accurate in terms of similarity.

The three corollaries stated below are formed based on Statement 1. The members (instance or datapoints) of each cluster are considered as a subset of features in the feature vector. The similarity of the features in a cluster is evaluated based on the correlation among the cluster members. Moreover, Corollary 3 states the nature of the redundant features with respect to formation of the cluster. For example, if two features $\left\{X_{1}, X_{2}\right\}$ are highly correlated, then the two features become redundant features since they have same information in terms of correlation measure. In other words, the correlation measure provides statistical association between any given a pair of features.

Corollary 1 The intra-cluster features are similar (highly correlated).

Corollary 2 The inter-cluster features are dissimilar (less correlated or uncorrelated).

Corollary 3 Similar features are redundant features (highly correlated).
Consider the features $X=\left\{X_{1}, X_{2}, X_{3}, \ldots, X_{N}\right\}$ of a dataset $D$. Let $K$ be the number of clusters to be formed from $D$, and $\mathrm{FC}=\left\{\mathrm{fc}_{1}, \mathrm{fc}_{2}, \ldots \mathrm{fc}_{k}\right\}$ is the set of clusters. Let $\varphi_{p}$ be the intra-cluster correlation of the $p$ th cluster $\mathrm{fc}_{p}$ represented as $\varphi_{p}\left(\mathrm{fc}_{p}\right)$. Then, the average intra-cluster correlation of $K$ number of clusters of $D$ is represented as $F C(D)$ as in Eq. (7).

$\mathrm{FC}(D)=\frac{1}{K} \sum_{p=1}^{K} \varphi_{p}\left(\mathrm{fc}_{p}\right)$

$\varphi_{p}\left(\mathrm{fc}_{p}\right)$ is calculated from the correlation CR between two features $X_{i}$ and $X_{j}$ within the $p$ th cluster using Eq. (8) where $m$ is the total number of features present in $p$ th cluster.

$\varphi_{p}\left(\mathrm{fc}_{p}\right) \frac{1}{m(m-1)} \sum_{X_{i}, X_{j} \in X} \mathrm{CR}_{X_{i}, X_{j}} \because i<j$

$\mathrm{CR}_{X_{i}, X_{j}}=\frac{1}{M-1} \sum_{\tau=1}^{M}\left(\frac{X_{i_{\tau}}-\mu_{X_{i}}}{\sigma_{X_{i}}}\right)\left(\frac{X_{j_{\tau}}-\mu_{X_{j}}}{\sigma_{X_{j}}}\right)$

The correlation CR between two features $X_{i}$ and $X_{j}$ is $\mathrm{CR}_{X_{i}, X_{j}}$ as defined in Eq. (9). The parameters $\mu_{X_{i}}$ and $\sigma_{X_{i}}$ are the mean and standard deviation of $X_{i} \cdot \mu_{X_{j}}$ and $\sigma_{X_{j}}$ are the mean and standard deviation of $X_{j} . M$ represents the total number of samples in the feature.

Consider the number of clusters $K=4$ and three different clustering methods $M_{1}, M_{2}, M_{3}$. The performance of these clustering methods is analyzed as follows:

Method $1\left(\mathrm{M}_{1}\right)$ If $\varphi_{1}\left(\mathrm{fc}_{1}\right)=\varphi_{2}\left(\mathrm{fc}_{2}\right)=\varphi_{3}\left(\mathrm{fc}_{3}\right)=\varphi_{4}\left(\mathrm{fc}_{4}\right)=1.0$ 0 , then $\mathrm{FC}(D)=1$.

Method $2\left(\mathrm{M}_{2}\right)$ If $\varphi_{1}\left(\mathrm{fc}_{1}\right)=\varphi_{2}\left(\mathrm{fc}_{2}\right)=\varphi_{3}\left(\mathrm{fc}_{3}\right)=\varphi_{4}\left(\mathrm{fc}_{4}\right)=0.5$ 0 , then $\mathrm{FC}(D)=0.5$.

Method $3\left(\mathrm{M}_{3}\right)$ If $\varphi_{1}\left(\mathrm{fc}_{1}\right)=\varphi_{2}\left(\mathrm{fc}_{2}\right)=\varphi_{3}\left(\mathrm{fc}_{3}\right)=\varphi_{4}\left(\mathrm{fc}_{4}\right)=0.2$

5 , then $\mathrm{FC}(D)=0.25$.

From Corollaries 1,2 and 3 with the sample performance of clustering methods $M_{1}, M_{2}$ and $M_{3}$ in terms of $\varphi_{p}\left(\mathrm{fc}_{p}\right)$ and $\mathrm{FC}(D)$, it is evident that the intra-cluster correlation of the features is directly proportional to the intra-cluster redundancy of the features and the Method 1 seems to perform better in grouping the redundant features since it exhibits higher $F C(D)$ value compared to other methods.

\section{Experimental setup and procedure}

The experiments are conducted using MATLAB12b with the system configuration of Intel ${ }^{\circledR}$ Core $^{\text {TM }} 2$ CPU T5300 @ $1.73 \mathrm{GHz}$ processor, 4 GB memory (RAM) and 32-bit 
Table 1 Details of the datasets

\begin{tabular}{|c|c|c|c|}
\hline Dataset & Features & Instances & Classes \\
\hline ORL10Pa & 10,304 & 100 & 10 \\
\hline $\mathrm{PIX} 10 \mathrm{P}^{\mathrm{a}}$ & 10,000 & 100 & 10 \\
\hline PIE10Pa & 2420 & 210 & 10 \\
\hline $\mathrm{AR} 10 \mathrm{P}^{\mathrm{a}}$ & 2400 & 130 & 10 \\
\hline $\mathrm{SRBCT}^{\mathrm{b}}$ & 2308 & 83 & 4 \\
\hline ORL_32 $\times 32^{c}$ & 1024 & 400 & 40 \\
\hline Yale_64 $\times 64^{c}$ & 4096 & 165 & 15 \\
\hline COIL20 & 1024 & 1440 & 20 \\
\hline DBWorld e-mails ${ }^{d}$ & 4702 & 64 & 2 \\
\hline
\end{tabular}

ahttps://featureselection.asu.edu/datasets/

bhttps://www.gems-system.org/

chttps://www.cad.zju.edu.cn/home/dengcai/Data/

${ }^{\mathrm{d}}$ https://archive.ics.uci.edu/ml/datasets/

Windows vista Home Premium Operating system. The performance of the clustering methods is tested on various high-dimensional datasets listed in Table 1. Further, the performance of the clustering methods $\mathrm{KC}, \mathrm{EC}$ and $\mathrm{HC}$ is tested in terms average intra-cluster redundancy rate and runtime.

The experiment is conducted with the following procedure: Initially, the dataset is given to the clustering method with the number of clusters to be formed $K$. Then the $K$ numbers of clusters are formed. The corresponding runtime is noted and the intra-cluster redundancy rate is calculated for all $K$ numbers of clusters for each dataset. The average intra-cluster redundancy rate is calculated by averaging intra-cluster redundancy rates from $K$ numbers of clusters. For this experiment, the average intra-cluster redundancy rate and runtime are obtained by varying the number of clusters $K$ from 2 to 10 .

\section{Experimental results}

The experimental results are obtained in terms of runtime and average intra-cluster redundancy rate with KC, EC and $\mathrm{HC}$ clustering techniques on all the datasets as listed in Table 1 with respect to number of clusters and tabulated from Tables 2, 3, 4, 5, 6, 7, 8, 9 and 10. The average runtime of $\mathrm{KC}, \mathrm{EC}$ and $\mathrm{HC}$ clustering techniques on all the datasets
Table 2 Runtime and average intra-cluster redundancy rate of $\mathrm{KC}, \mathrm{EC}$ and $\mathrm{HC}$ on the dataset PIE10P with respect to number of clusters
Table 3 Runtime and average intra-cluster redundancy rate of $\mathrm{KC}, \mathrm{EC}$ and $\mathrm{HC}$ on the dataset AR10P with respect to number of clusters

\begin{tabular}{|c|c|c|c|c|c|c|}
\hline \multirow[t]{2}{*}{$\begin{array}{l}\text { Number of } \\
\text { clusters }\end{array}$} & \multicolumn{3}{|c|}{ Runtime } & \multicolumn{3}{|c|}{$\begin{array}{l}\text { Average intra-cluster redundancy } \\
\text { rate }\end{array}$} \\
\hline & $\mathrm{KC}$ & $\mathrm{EC}$ & $\mathrm{HC}$ & $\mathrm{KC}$ & $\mathrm{EC}$ & $\mathrm{HC}$ \\
\hline 2 & 0.510 & 4.278 & 40.885 & 0.558 & 0.513 & 0.205 \\
\hline 3 & 0.460 & 5.883 & 40.466 & 0.588 & 0.551 & 0.136 \\
\hline 4 & 0.452 & 1.225 & 40.551 & 0.600 & 0.596 & 0.102 \\
\hline 5 & 0.737 & 9.820 & 40.046 & 0.632 & 0.643 & 0.082 \\
\hline 6 & 1.640 & 5.394 & 39.575 & 0.643 & 0.637 & 0.139 \\
\hline 7 & 0.814 & 1.604 & 40.391 & 0.665 & 0.663 & 0.222 \\
\hline 8 & 4.416 & 4.635 & 39.802 & 0.669 & 0.663 & 0.246 \\
\hline 9 & 1.888 & 6.303 & 40.535 & 0.659 & 0.700 & 0.250 \\
\hline 10 & 1.105 & 5.717 & 39.488 & 0.674 & 0.711 & 0.226 \\
\hline
\end{tabular}

\begin{tabular}{|c|c|c|c|c|c|c|}
\hline \multirow[t]{2}{*}{$\begin{array}{l}\text { Number of } \\
\text { clusters }\end{array}$} & \multicolumn{3}{|c|}{ Runtime } & \multicolumn{3}{|c|}{$\begin{array}{l}\text { Average intra-cluster redundancy } \\
\text { rate }\end{array}$} \\
\hline & $\mathrm{KC}$ & $\mathrm{EC}$ & $\mathrm{HC}$ & $\overline{\mathrm{KC}}$ & EC & $\mathrm{HC}$ \\
\hline 2 & 0.418 & 7.061 & 39.338 & 0.324 & 0.370 & 0.421 \\
\hline 3 & 0.403 & 6.271 & 37.964 & 0.413 & 0.399 & 0.470 \\
\hline 4 & 0.346 & 9.112 & 37.880 & 0.476 & 0.467 & 0.482 \\
\hline 5 & 0.542 & 13.794 & 39.316 & 0.533 & 0.475 & 0.512 \\
\hline 6 & 0.416 & 6.873 & 37.896 & 0.552 & 0.485 & 0.545 \\
\hline 7 & 0.652 & 12.029 & 38.880 & 0.567 & 0.509 & 0.598 \\
\hline 8 & 0.971 & 12.922 & 38.685 & 0.592 & 0.505 & 0.613 \\
\hline 9 & 1.887 & 13.271 & 37.367 & 0.601 & 0.560 & 0.613 \\
\hline 10 & 5.242 & 4.362 & 38.104 & 0.599 & 0.587 & 0.590 \\
\hline
\end{tabular}


Table 4 Runtime and average intra-cluster redundancy rate of $\mathrm{KC}, \mathrm{EC}$ and $\mathrm{HC}$ on the dataset SRBCT with respect to number of clusters
Table 5 Runtime and average intra-cluster redundancy rate of $\mathrm{KC}, \mathrm{EC}$ and $\mathrm{HC}$ on the dataset ORL_32 332 with respect to number of clusters

\begin{tabular}{|c|c|c|c|c|c|c|}
\hline \multirow[t]{2}{*}{$\begin{array}{l}\text { Number of } \\
\text { clusters }\end{array}$} & \multicolumn{3}{|c|}{ Runtime } & \multicolumn{3}{|c|}{$\begin{array}{l}\text { Average intra-cluster redundancy } \\
\text { rate }\end{array}$} \\
\hline & $\mathrm{KC}$ & EC & $\mathrm{HC}$ & $\mathrm{KC}$ & $\mathrm{EC}$ & $\mathrm{HC}$ \\
\hline 2 & 0.404 & 1.927 & 35.105 & 0.170 & 0.160 & 0.291 \\
\hline 3 & 0.163 & 5.807 & 34.633 & 0.195 & 0.224 & 0.332 \\
\hline 4 & 1.156 & 4.792 & 35.279 & 0.196 & 0.205 & 0.332 \\
\hline 5 & 0.908 & 9.978 & 34.486 & 0.200 & 0.172 & 0.352 \\
\hline 6 & 0.593 & 9.797 & 34.510 & 0.202 & 0.207 & 0.330 \\
\hline 7 & 1.935 & 10.120 & 34.537 & 0.201 & 0.220 & 0.283 \\
\hline 8 & 9.402 & 25.505 & 35.089 & 0.234 & 0.218 & 0.196 \\
\hline 9 & 5.961 & 10.925 & 34.623 & 0.237 & 0.190 & 0.229 \\
\hline 10 & 4.017 & 19.577 & 34.125 & 0.258 & 0.189 & 0.207 \\
\hline
\end{tabular}

\begin{tabular}{|c|c|c|c|c|c|c|}
\hline \multirow[t]{2}{*}{$\begin{array}{l}\text { Number of } \\
\text { clusters }\end{array}$} & \multicolumn{3}{|c|}{ Runtime } & \multicolumn{3}{|c|}{$\begin{array}{l}\text { Average intra-cluster redundancy } \\
\text { rate }\end{array}$} \\
\hline & $\mathrm{KC}$ & $\mathrm{EC}$ & $\mathrm{HC}$ & $\mathrm{KC}$ & $\mathrm{EC}$ & $\mathrm{HC}$ \\
\hline 2 & 0.420 & 0.719 & 0.308 & 0.308 & 0.316 & 0.284 \\
\hline 3 & 1.222 & 0.596 & 0.309 & 0.309 & 0.308 & 0.433 \\
\hline 4 & 2.266 & 0.806 & 0.365 & 0.365 & 0.373 & 0.415 \\
\hline 5 & 1.123 & 0.986 & 0.380 & 0.380 & 0.348 & 0.439 \\
\hline 6 & 1.165 & 1.206 & 0.419 & 0.419 & 0.456 & 0.458 \\
\hline 7 & 2.131 & 1.434 & 0.432 & 0.432 & 0.424 & 0.470 \\
\hline 8 & 1.899 & 1.662 & 0.445 & 0.445 & 0.453 & 0.504 \\
\hline 9 & 1.881 & 1.837 & 0.491 & 0.491 & 0.483 & 0.507 \\
\hline 10 & 2.927 & 2.019 & 0.486 & 0.486 & 0.460 & 0.513 \\
\hline
\end{tabular}

Table 6 Runtime and average intra-cluster redundancy rate of $\mathrm{KC}, \mathrm{EC}$ and $\mathrm{HC}$ on the dataset Yale_64×64 with respect to number of clusters

\begin{tabular}{|c|c|c|c|c|c|c|}
\hline \multirow[t]{2}{*}{$\begin{array}{l}\text { Number of } \\
\text { clusters }\end{array}$} & \multicolumn{3}{|c|}{ Runtime } & \multicolumn{3}{|c|}{$\begin{array}{l}\text { Average intra-cluster redundancy } \\
\text { rate }\end{array}$} \\
\hline & $\mathrm{KC}$ & $\mathrm{EC}$ & $\mathrm{HC}$ & $\overline{\mathrm{KC}}$ & $\mathrm{EC}$ & $\mathrm{HC}$ \\
\hline 2 & 0.699 & 2.338 & 118.586 & 0.294 & 0.291 & 0.308 \\
\hline 3 & 0.904 & 24.805 & 119.030 & 0.322 & 0.325 & 0.480 \\
\hline 4 & 2.318 & 8.636 & 119.271 & 0.374 & 0.407 & 0.533 \\
\hline 5 & 1.420 & 30.768 & 119.170 & 0.424 & 0.424 & 0.596 \\
\hline 6 & 4.206 & 41.496 & 118.679 & 0.429 & 0.389 & 0.618 \\
\hline 7 & 2.199 & 28.118 & 117.801 & 0.491 & 0.442 & 0.642 \\
\hline 8 & 4.901 & 90.846 & 118.388 & 0.472 & 0.493 & 0.667 \\
\hline 9 & 3.639 & 34.078 & 115.786 & 0.501 & 0.491 & 0.686 \\
\hline 10 & 3.457 & 30.123 & 115.112 & 0.515 & 0.475 & 0.672 \\
\hline
\end{tabular}

with respect to number of clusters is illustrated in Fig. 1, and the mean value of average redundancy rate of intraclusters formed by KC, EC and $\mathrm{HC}$ clustering techniques on all the datasets with respect to number of clusters is illustrated in Fig. 2.

\subsection{Discussion}

From the experimental results (Tables 2, 3, 4, 5, 6, 7, 8, 9 and 10), it is obvious that the KC cluster takes lesser runtime compared to $\mathrm{EC}$ and $\mathrm{HC}$ clustering techniques. From Table 2, it is observed that KC and EC clusters produce higher average intra-cluster redundancy rate compared to HC for PIE10P dataset. As seen from Table 3, the three 
Table 7 Runtime and average intra-cluster redundancy rate of $\mathrm{KC}, \mathrm{EC}$ and $\mathrm{HC}$ on the dataset COIL20 with respect to number of clusters
Table 8 Runtime and average intra-cluster redundancy rate of $\mathrm{KC}, \mathrm{EC}$ and $\mathrm{HC}$ on the respect to number of clusters dataset DBWorld e-mails with

\begin{tabular}{llllllll}
\hline $\begin{array}{l}\text { Number of } \\
\text { clusters }\end{array}$ & \multicolumn{2}{l}{ Runtime } & & & \multicolumn{3}{l}{$\begin{array}{l}\text { Average intra-cluster redundancy } \\
\text { rate }\end{array}$} \\
\cline { 2 - 3 } & KC & EC & HC & & KC & EC & HC \\
\hline 2 & 0.902 & 4.633 & 11.165 & & 0.273 & 0.249 & 0.280 \\
3 & 0.825 & 6.512 & 12.798 & & 0.295 & 0.287 & 0.401 \\
4 & 1.829 & 9.452 & 12.261 & & 0.364 & 0.398 & 0.460 \\
5 & 2.354 & 11.219 & 11.991 & & 0.397 & 0.398 & 0.454 \\
6 & 7.148 & 14.834 & 11.391 & & 0.373 & 0.402 & 0.449 \\
7 & 2.313 & 15.581 & 11.809 & & 0.470 & 0.422 & 0.486 \\
8 & 4.276 & 17.205 & 11.044 & & 0.451 & 0.428 & 0.498 \\
9 & 8.505 & 19.606 & 10.907 & & 0.460 & 0.431 & 0.525 \\
10 & 4.427 & 22.531 & 10.304 & & 0.488 & 0.430 & 0.514 \\
\hline
\end{tabular}

\begin{tabular}{|c|c|c|c|c|c|c|}
\hline \multirow[t]{2}{*}{$\begin{array}{l}\text { Number of } \\
\text { clusters }\end{array}$} & \multicolumn{3}{|c|}{ Runtime } & \multicolumn{3}{|c|}{$\begin{array}{l}\text { Average intra-cluster redundancy } \\
\text { rate }\end{array}$} \\
\hline & $\mathrm{KC}$ & $\mathrm{EC}$ & $\mathrm{HC}$ & $\mathrm{KC}$ & $\mathrm{EC}$ & $\mathrm{HC}$ \\
\hline 2 & 0.809 & 1.842 & 147.767 & 0.104 & 0.087 & 0.110 \\
\hline 3 & 0.177 & 2.691 & 146.416 & 0.224 & 0.201 & 0.141 \\
\hline 4 & 0.264 & 0.575 & 146.283 & 0.282 & 0.304 & 0.168 \\
\hline 5 & 0.892 & 0.524 & 146.810 & 0.315 & 0.289 & 0.169 \\
\hline 6 & 0.409 & 0.435 & 147.056 & 0.288 & 0.402 & 0.143 \\
\hline 7 & 0.763 & 4.351 & 148.387 & 0.336 & 0.369 & 0.140 \\
\hline 8 & 0.669 & 5.769 & 147.273 & 0.401 & 0.283 & 0.154 \\
\hline 9 & 0.986 & 3.516 & 153.320 & 0.434 & 0.308 & 0.137 \\
\hline 10 & 1.080 & 1.585 & 151.871 & 0.350 & 0.300 & 0.157 \\
\hline
\end{tabular}

Table 9 Runtime and average intra-cluster redundancy rate of $\mathrm{KC}, \mathrm{EC}$ and $\mathrm{HC}$ on the dataset ORL10P with respect to number of clusters ${ }^{*}$ denotes the buffer overflow)

\begin{tabular}{|c|c|c|c|c|c|c|}
\hline \multirow[t]{2}{*}{$\begin{array}{l}\text { Number of } \\
\text { clusters }\end{array}$} & \multicolumn{3}{|c|}{ Runtime } & \multicolumn{3}{|c|}{$\begin{array}{l}\text { Average intra-cluster redundancy } \\
\text { rate }\end{array}$} \\
\hline & $\mathrm{KC}$ & $\mathrm{EC}$ & $\mathrm{HC}$ & $\mathrm{KC}$ & $\mathrm{EC}$ & $\mathrm{HC}$ \\
\hline 2 & 0.522 & 12.631 & $*$ & 0.287 & 0.302 & * \\
\hline 3 & 1.874 & 40.159 & * & 0.295 & 0.358 & * \\
\hline 4 & 10.502 & 77.960 & * & 0.335 & 0.351 & * \\
\hline 5 & 5.556 & 107.354 & * & 0.351 & 0.344 & $*$ \\
\hline 6 & 3.775 & 169.686 & * & 0.375 & 0.372 & $*$ \\
\hline 7 & 7.816 & 209.701 & * & 0.417 & 0.382 & * \\
\hline 8 & 6.889 & 258.095 & * & 0.428 & 0.386 & * \\
\hline 9 & 5.775 & 185.397 & * & 0.442 & 0.393 & * \\
\hline 10 & 24.168 & 136.445 & * & 0.468 & 0.415 & * \\
\hline
\end{tabular}

clustering techniques perform equally in terms of average intra-cluster redundancy rate for the dataset AR10P. For the dataset SRBCT, KC and EC clusters yield better average intra-cluster redundancy rate (Table 4). Also, $\mathrm{HC}$ performs better in terms of average intra-cluster redundancy rate for the dataset ORL_ $32 \times 32$ as shown in Table 5 .

From Table 6 , it is observed that $\mathrm{HC}$ results in higher average intra-cluster redundancy rate for the dataset
Yale_64 $\times 64$. For the COIL20 dataset (Table 7), all the three clustering techniques yield more or less similar average intra-cluster redundancy rate. Table 8 depicts that $\mathrm{KC}$ and EC exhibit higher average intra-cluster redundancy rate than $\mathrm{HC}$ for the dataset DBWorld e-mails. KC and EC yield better average intra-cluster redundancy rate for the dataset ORL10P. However, HC undergoes buffer overflow due to the high-dimensional space for the dataset ORL10P 
Table 10 Runtime and average intra-cluster redundancy rate of $\mathrm{KC}, \mathrm{EC}$ and $\mathrm{HC}$ on the dataset PIX10P with respect to number of clusters $\left({ }^{*}\right.$ denotes the buffer overflow)

\begin{tabular}{|c|c|c|c|c|c|c|}
\hline \multirow[t]{2}{*}{$\begin{array}{l}\text { Number of } \\
\text { clusters }\end{array}$} & \multicolumn{3}{|c|}{ Runtime } & \multicolumn{3}{|c|}{$\begin{array}{l}\text { Average intra-cluster redundancy } \\
\text { rate }\end{array}$} \\
\hline & $\mathrm{KC}$ & $\mathrm{EC}$ & $\mathrm{HC}$ & $\mathrm{KC}$ & $\mathrm{EC}$ & $\mathrm{HC}$ \\
\hline 2 & 0.456 & 14.480 & * & 0.390 & 0.450 & $*$ \\
\hline 3 & 1.513 & 25.455 & * & 0.452 & 0.425 & * \\
\hline 4 & 1.399 & 26.395 & * & 0.480 & 0.436 & * \\
\hline 5 & 1.374 & 27.337 & * & 0.492 & 0.494 & * \\
\hline 6 & 2.016 & 142.461 & * & 0.574 & 0.475 & * \\
\hline 7 & 2.851 & 103.080 & * & 0.598 & 0.475 & * \\
\hline 8 & 5.482 & 184.380 & * & 0.632 & 0.477 & * \\
\hline 9 & 2.652 & 225.344 & * & 0.616 & 0.492 & * \\
\hline 10 & 2.930 & 124.827 & * & 0.650 & 0.496 & * \\
\hline
\end{tabular}

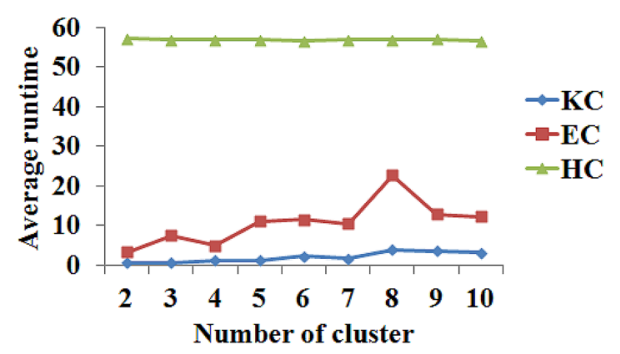

Fig. 1 Average runtime of $\mathrm{KC}, \mathrm{EC}$, and $\mathrm{HC}$ on all the datasets except ORL10P and PIX10P (since HC induces buffer overflow for the datasets ORL10P and PIX10P) with respect to number of clusters

(Table 9). KC yields higher average intra-cluster redundancy rate than EC and $\mathrm{HC}$ experiences buffer overflow for the dataset PIX10P as it is evident from Table 10.

From Figs. 1 and 2, it is apparent that KC takes lesser average runtime and produces higher mean value of average intra-cluster redundancy rate than EC and $\mathrm{HC}$ on all the datasets except ORL10P and PIX10P since HC induces buffer overflow for the datasets ORL10P and PIX10P.

Further, it is observed that the $\mathrm{HC}$ takes more time to form clusters due to the inherent computational complexity and it exhibits poor performance in terms of overall intra-cluster redundancy rate compared to EC and KC. Further, $\mathrm{HC}$ induces buffer overflow when the number of features is more (high-dimensional data) due to high space complexity. Therefore, $\mathrm{HC}$ is not a suitable choice for redundancy analysis in high-dimensional space. EC cluster has more computational complexity than $\mathrm{KC}$ and its overall performance in terms of intra-cluster redundancy rate is better than HC. Nevertheless, its performance is poor when compared to KC. KC clustering technique performs better in redundancy analysis since it produces overall higher intra-cluster redundancy rate and takes less computational time compared to EC and HC. Therefore, it is concluded that $\mathrm{KC}$ clustering technique can be the best

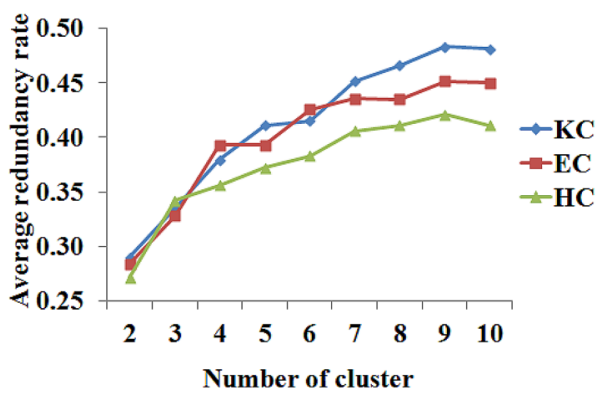

Fig. 2 Mean value of average intra-cluster redundancy rate of $\mathrm{KC}$, $\mathrm{EC}$, and $\mathrm{HC}$ on all the datasets except ORL10P and PIX10P (since HC induces buffer overflow for the datasets ORL10P and PIX10P) with respect to number of clusters

choice for redundancy analysis for the high-dimension data.

\section{Conclusion}

This paper presented an empirical study on various clustering techniques for redundancy analysis in feature selection for high-dimensional data classification. The performance of these clustering approaches, namely centroid-based K-means clustering, distribution-based expectation maximization clustering and connectivebased hierarchical clustering, is evaluated in terms of runtime and average intra-cluster redundancy rate. From the results, it is observed that the K-means clustering is suitable for redundancy analysis in feature selection since it yields higher intra-cluster redundancy rate and takes less runtime for the high-dimensional space. Therefore, it is suggested that the K-means clustering can be a better choice for redundancy analysis phase of the feature selection process for the high-dimensional data 
classification. Moreover, this work may be extended with different types of clustering techniques and various statistical measures may be adopted for performance evaluation. Furthermore, this redundancy analysis method can be combined with any one of the relevancy analysis method for selecting the significant features from the high-dimensional data to achieve higher accuracy for the classification tasks for various applications.

\section{Compliance with ethical standards}

Conflict of interest The authors declare that they have no conflict of interest.

\section{References}

1. Yoo l et al (2011) Data mining in healthcare and biomedicine: a survey of the literature. J Med Syst 36(4):2431-2448

2. Singh DAAG, Balamurugan S, Leavline EL (2012) An empirical study on dimensionality reduction and improvement of classification accuracy using feature subset selection and ranking. In: Proceedings of IEEE international conference on emerging trends in science, engineering and technology (INCOSET), pp 102-108

3. Liu D, Qian H, Dai G, Zhang Z (2013) An iterative SVM approach to feature selection and classification in high-dimensional datasets. Pattern Recognit 46(9):2531-2537

4. Imoto S, Miyano S (2012) A top-r feature selection algorithm for microarray gene expression data. IEEE/ACM Trans Comput Biol Bioinform 9(3):754-764

5. Şen B, Peker M, Çavuşoğlu A, Çelebi FV (2014) A comparative study on classification of sleep stage based on EEG signals using feature selection and classification algorithms. J Med Syst 38(18):1-21

6. Liao S-H, Chu P-H, Hsiao P-Y (2012) Data mining techniques and applications-a decade review from 2000 to 2011. Expert Syst Appl 39(12):11303-11311

7. Celebi ME, Kingravi HA, Vela PA (2013) A comparative study of efficient initialization methods for the k-means clustering algorithm. Expert Syst Appl 40(1):200-210

8. Han J, Kamber M, Pei J (2011) Data mining: concepts and techniques, Third edition (the Morgan Kaufmann series in data management systems), 3rd edn. Morgan Kaufmann Publishers Inc, Amsterdam

9. McLachlan GJ (1997) EM algorithm and extensions. Wiley, New York

10. Gan G, Ma C, Wu J (2007) Data clustering: theory, algorithms, and applications. Society for Industrial and Applied Mathematics, Philadelphia

11. Gürdür D, El-khoury J, Törngren M (2019) Digitalizing Swedish industry: What is next? Data analytics readiness assessment of Swedish industry, according to survey results. Comput Ind 105:153-163

12. Zhaoa L, Youb F (2019) A data-driven approach for industrial utility systems optimization under uncertainty. Energy 182:559-569

13. Abpeykara S, Ghateea M, Zareb H (2019) Ensemble decision forest of RBF networks via hybrid feature clustering approach for high-dimensional data classification. Comput Stat Data Anal 131:12-36
14. Singh DAAG, Leavline EJ (2013) Data mining in network security-techniques and tools: a research perspective. JTheor Appl Inf Technol 57(2):269-278

15. Singh DAAG, Priyadharshini R, Leavline EJ (2018) Cuckoo optimisation based intrusion detection system for cloud computing. Int J Comput Netw Inf Secur (IJCNIS) 10(11):42-49. https://doi. org/10.5815/ijcnis.2018.11.05

16. Zhang Z, Wang C, Gao Y, Chen Y, Chen J (2020) Passenger flow forecast of rail station based on multi-source data and long short term memory network. IEEE Access 8:28475-28483. https ://doi.org/10.1109/ACCESS.2020.2971771

17. Arora N, DeepKaur P (2020) A Bolasso based consistent feature selection enabled random forest classification algorithm: an application to credit risk assessment. Appl Soft Comput 86:105936

18. Ravisankara P, Ravia V, Raghava Raoa G, Boseb I (2011) Detection of financial statement fraud and feature selection using data mining techniques. Decis Support Syst 50(2):491-500

19. Saqlain SM, Sher M, Shah FA et al (2019) Fisher score and Matthews correlation coefficient-based feature subset selection for heart disease diagnosis using support vector machines. Knowl Inf Syst 58:139-167. https://doi.org/10.1007/s10115-018-1185-y

20. Singh DAAG, Krishna Kumari B, Leavline EJ (2017) Firefly optimization based dimensionality reduction for improving accuracy in job hunting. Indian J Comput Sci Eng (IJCSE) 8(3):346-357

21. Maya Gopal PS, Bhargavi R (2019) Performance evaluation of best feature subsets for crop yield prediction using machine learning algorithms. Appl Artif Intell 33(7):621-642. https://doi. org/10.1080/08839514.2019.1592343

22. Singh DAAG, Leavline EJ, Abirami AK, Dhivya M (2018) Plant disease detection system using bag of visual words. IJ Inf Technol Comput Sci 8(9):57-63. https://doi.org/10.5815/ijitcs.2018.09.07

23. Kumar S, Sharma B, Sharma VK, Sharma H, Bansal JC (2018) Plant leaf disease identification using exponential spider monkey optimization. Sustain Comput Inform Syst. https://doi. org/10.1016/j.suscom.2018.10.004

24. Singh DAAG, Leavline EJ, Priyanka V, Swathi V (2016) Agriculture classification system using differential evolution algorithm. Int Adv Res J Sci Eng Technol 3(5):24-28

25. Singh DAAG, Fernando AE, Leavline EJ (2016) Experimental study on feature selection methods for software fault detection. In: 2016 International conference on circuit, power and computing technologies (ICCPCT). Nagercoil, pp 1-6. https:// doi.org/10.1109/ICCPCT.2016.7530156

26. Singh AAGD, Fernando EA, Leavline EJ (2016) Software Fault Detection using Honey Bee Optimization. Int J Appl Inf Syst 11(1):1-9

27. Lian J, Dong P, Zhang Y, Pan J, Liu K (2020) A novel data-driven tropical cyclone track prediction model based on CNN and GRU with multi-dimensional feature selection. IEEE Access 8:9711497128. https://doi.org/10.1109/ACCESS.2020.2992083

28. Patel SP, Upadhyay SH (2020) Euclidean distance based feature ranking and subset selection for bearing fault diagnosis. Expert Syst Appl 154:113400

29. Mlakar U, Fister I, Brest J, Potočnik B (2017) Multi-objective differential evolution for feature selection in facial expression recognition systems. Expert Syst Appl 89:129-137

30. Liu ZT, Wu M, Cao WH, Mao JW, Xu JP, Tan GZ (2018) Speech emotion recognition based on feature selection and extreme learning machine decision tree. Neurocomputing 273:271-280

31. Özseven T (2019) A novel feature selection method for speech emotion recognition. Appl Acoust 146:320-326

32. El Barbary OG, Salama AS (2018) Feature selection for document classification based on topology. Egypt Inform J 19(2):129-132 
33. Agnihotria D, Vermaa K, Tripathib P (2017) Variable global feature selection scheme for automatic classification of text documents. Expert Syst Appl 81:268-281

34. Kuang H, Chen L, Chan LLH, Cheung RCC, Yan H (2018) Feature selection based on tensor decomposition and object proposal for night-time multiclass vehicle detection. IEEE Trans Syst Man Cybern Syst 49(1):71-80. https://doi.org/10.1109/ TSMC.2018.2872891
Publisher's Note Springer Nature remains neutral with regard to jurisdictional claims in published maps and institutional affiliations.

\section{SN Applied Sciences}

